\title{
Checklist of liverworts of the Lapland State Nature Biosphere Reserve (Murmansk Province, Russia)
}

\author{
Eugene A. Borovichev ${ }^{1,2}$ \\ ${ }^{1}$ N.A. Avrorin Polar-Alpine Botanical Garden-Institute of Kola Science Centre RAS, Apatity, 184209, Russia; \\ ${ }^{2}$ Institute of the Industrial Ecology Problems of the North of the Kola Science Center of RAS, Apatity, 184209, Russia \\ E-mail: borovichyok@mail.ru
}

\begin{abstract}
This new list of hepatics for the Lapland State Nature Biosphere Reserve (LSNBR), one of the largest reserves in European Russia, consists of 173 species. Here are summarized all available data on the diversity of liverworts of LSNBR, including approximately 2500 specimens collected by the author. The liverwort flora is the richest amongst those of large State Nature Reserves of the European part of Russia. Eight taxa of liverworts are known in Murmansk Province from the LSNBR only: Frullania tamarisci, Lejeunea cavifolia, Lophoziopsis excisa var. elegans, Mannia triandra, Reboulia hemisphaerica, Oleolophozia perssonii, Nowellia curvifolia, Scapania calcicola. The liverwort diversity varies slightly between different LSNBR Mountains: in Salnye Tundry Mts there were recorded 139 species, in Chuna-tundra Mts - 133, in Monche-tundra Mts - 124, in Nyavka-tundra - 112. The territory gives a good representation for protection and sustainable development of rare hepatic species - fifteen species are red-listed in Europe, four liverworts are included in the Red Data Book of the Russian Federation and thirty one taxa are protected in Murmansk Province.
\end{abstract}

\section{INTRODUCTION}

The Lapland State Nature Biosphere Reserve (LSNBR) is situated in the north-west of Russia, in the central part of Murmansk Province (Fig. 1) between $67^{\circ} 39^{\prime}-68^{\circ} 15^{\prime} \mathrm{N}$ and $31^{\circ} 10^{\prime}-32^{\circ} 45^{\prime} \mathrm{E}$. LSNBR is one of the largest reserves in European Russia; the protected area occupies $2784 \mathrm{~km}^{2}$. The relief of the Reserve is of very rugged mountains and undulating topography. As a result of the most recent glaciation, the geomorphological features of mountains of the LSNBR include flat surfaces, gentle slopes and well-developed lateral moraines. Absolute elevations of the Reserve varies from $103 \mathrm{~m}$ alt. (valleys of the Konya and Berezovaya Rivers) to $1114 \mathrm{~m}$ alt. (Ebrchorr Mt.). A considerable part of the territory is occupied by mountain tundra and includes four large mountain massifs, dissimilar in geomorphology - Salnye Tundry Mts, Monche-tundra Mts, Chuna-tundra Mts, Nyavka-tundra Mts.

The Salnye Tundry Mts are located in the northwest of the territory; maximum elevations are below $1000 \mathrm{~m}$ alt. - Elgoras Mt (997.3 m alt.) and Vuim Mt (990.2 m alt.). The Monche-tundra Mts are a ridge extending along the eastern boundary of the LSNBR, with the main peak Khipik Mt (965.3 m alt.). The Chuna-tundra Mts form a meridionally-orientated ridge which extends to the southern border of the LSNBR, with several peaks exceeding $1000 \mathrm{~m}$ alt. - Ebrchorr Mt

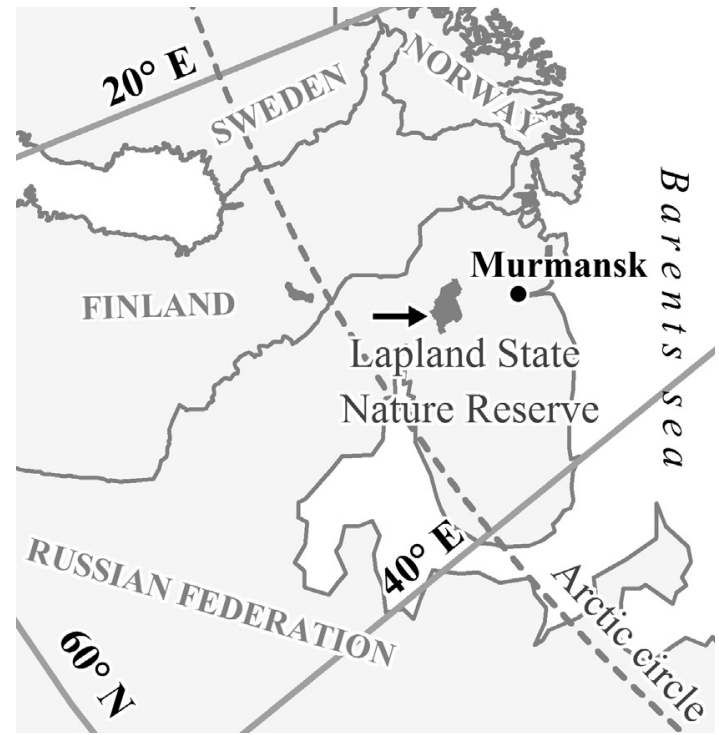

Fig. 1. The location of the Lapland State Nature Biosphere Reserve, Murmansk Province, Russia.

(1114 m alt.), Namlagchorr Mt (1064.8 m alt.). The central part of the Reserve is occupied by the Nyavka-tundra Mts, composed of several mountains with elevations below $700 \mathrm{~m}$ alt. (the highest point is Kreps Mt $697 \mathrm{~m}$ alt.) separated by narrow river valleys. South-western and 
southern parts of the Reserve are occupied by more or less planar and hilly landscapes with low elevations, rarely towering above the forest, with extensive forests and wetland territories.

The climate is suboceanic, being influenced by the north Atlantic and the Polar arctic front. Due to the Gulf Stream, the winter temperatures are relatively high preventing the development of permafrost. The climate is characterized by relatively mild winters and cool short summers; however, there may brief spells with negative temperatures in any summer month. The mean annual precipitation is relatively low, 330-445 $\mathrm{mm}$, much of which falls on the summer months. The snow cover normally lies from mid-October to the end of May-June; both its distribution and the time of melting in the mountains depend on the relief (Semyonov-Tyan-Schanskiy, 1988). One of the main soil-forming material types, covering large areas, are moraine deposits.

The Reserve is referred to the biogeographic provinces of the Lapponia tulomensis and Lapponia Imandrae (Heikinheimo \& Raatikainen, 1971; Uotila, 2013). The LSNBR is situated in the Northern boreal zone according to Ahti $\&$ al. (1968). All mountains carry distinguishable vertical vegetation zones. The foothills are covered by woodland. Higher up, at 300-450 m alt., is crooked birch forest wedging out on steeper slopes. The upper part of the slopes (over $400-450 \mathrm{~m}$ alt) and summits are occupied by mountain tundra with vast rock fields, detritus and bedrock outcrops.

\section{Exploration of the hepatic flora of the LSNBR}

Studying of liverwort flora of the LSNBR was started by N.M. Pushkina in the 1930's. The first list of bryophytes of the LSNBR was published in 1960 (Pushkina, 1960) and included 29 liverworts. Later, during ecological field work, E.N. Andrejeva investigated the structure of the moss layer in plant communities influenced by aerotechnogenic pollution from Severonikel smelter in Salnye, Monche- and Chuna-tundra Mts (Andrejeva, 1990, 2005) and listed 54 species in geobotanical tables. She recorded there new species for the LSNBR (Cephaloziella spinigera, Haplomitrium hookeri, Jungermannia pumila, Liochlaena lanceolata, Lophozia ascendens, Riccardia multifida). Most of them have been re-collected in the course of our investigations. After a short-term field trip of N.A. Konstantinova in the Salnye Tundry in 1993, the list of liveworts for the LSNBR increased to 82 species (Konstantinova, 2001). In 2001 and 2002, V.A. Bakalin observed liverworts of the Chuna-, Monche-tundra and Salnye tundra Mts. Therefore, at the beginning of our study in 2004, the liverwort flora numbered 116 species (Bakalin, 2004; Andrejeva, 2005).

\section{MATERIALS AND METHODS}

Materials were collected by the author in 2004-2011 during 8 field seasons in different areas of the LSNBR, and included the results of identification of some other collections and generalization of published data. The present report is based on: ca. 1000 specimens collected in Salnye Tundry Mts in 2004-2008; ca. 450 specimens in Monche-tundra Mts in 2009, 2011; ca. 850 specimens in Chuna-tundra Mts in 2005-2006, 2008-2010 and ca. 300 specimens in Nyavka-tundra Mts in 2007-2008. The total number of specimens collected by the author at 25 sites is about 2500 (fig. 2). Furthermore, there have been examined or identified a number of specimens gathered earlier by N.M. Pushkina, N.A. Konstantinova, E.N. Andreyeva, V.A. Bakalin, A.V. Melekhin, T.V. Chernenkova and N.Yu. Natsvaladze. Specimens are deposited in herbarium of the Polar-Alpine Botanical Garden (KPABG).

\section{RESULTS \\ List of species}

The taxa are arranged in alphabetical order. The names follow Konstantinova et al. (2009) with some updates from recent literature (Söderström et al., 2010; Vilnet et al., 2011, 2012; Rubasinghe et al., 2011a,b; Schill \& al., 2010; Váňa et al., 2012, 2013). Common synonyms are given in parenthesis. In terms of frequency, the liverworts in the LSNBR are arranged as follows: frequent (species collected more than 30 times), not rare (16-30 sites), sporadic (6-15 sites), rare (3-5 sites) and unique (found 1-2 times). The name of the area where a species was collected was marked as follows: ST - Salnye Tundry Mts; ChT - Chuna-Tundra Mts; MT - Monche-Tundra Mts; NT - Nyavka-Tundra Mts. After the Mts abbreviation there is given the number of areas where species were collected (1-25, cf. Fig. 2). 


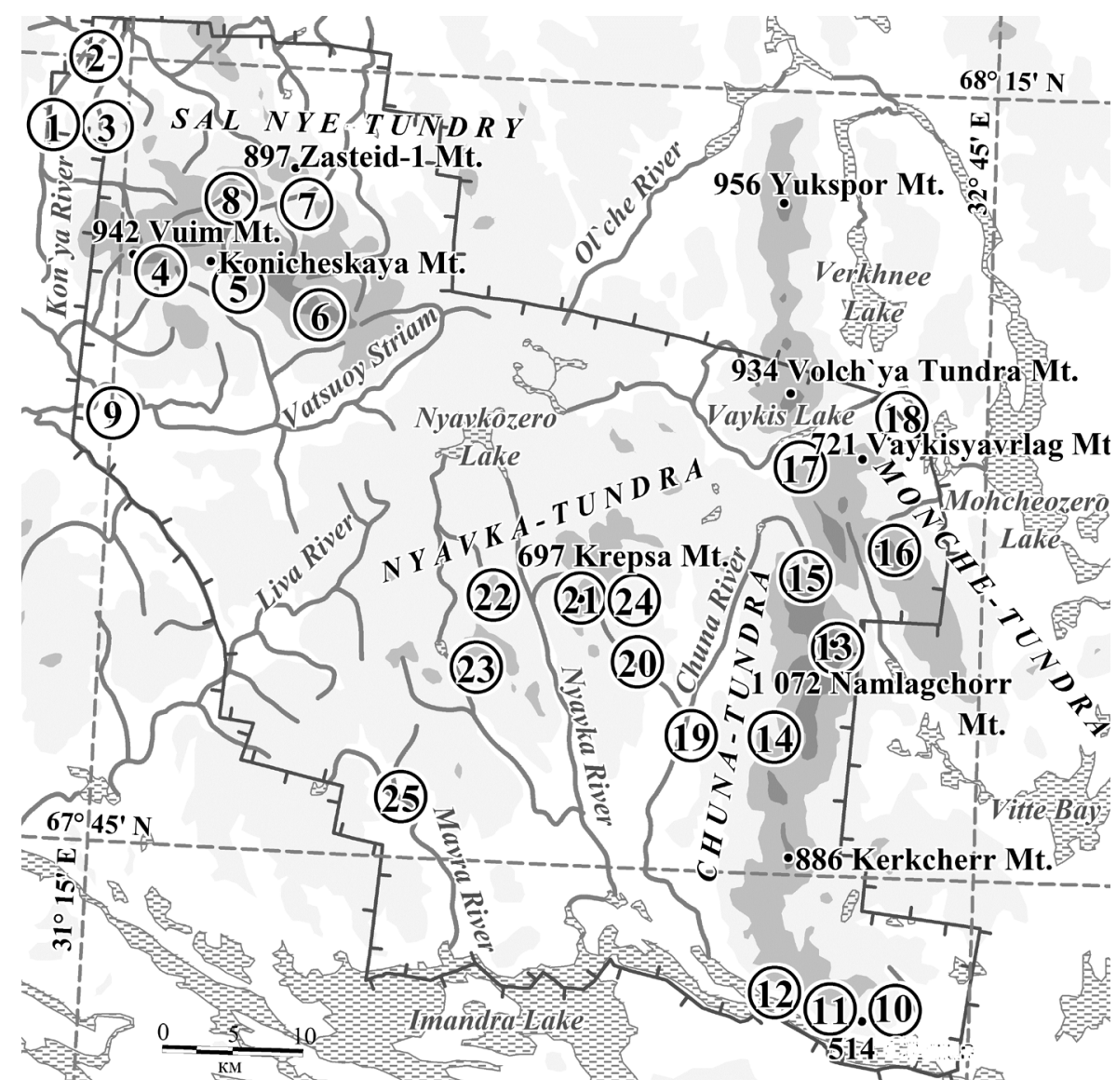

Fig. 2. Collecting localities. Salnye Tundry Mts. (ST): 1 . Pusozero Lake, $68^{\circ} 08^{\prime} \mathrm{N} 31^{\circ} 04^{\prime} \mathrm{E} ; 2$. Konya River Valley, $68^{\circ} 15^{\prime} \mathrm{N} 31^{\circ} 15^{\prime} \mathrm{E}$; 3. Pus and Pusik Mts, the canyon between them, $68^{\circ} 11^{\prime} \mathrm{N} 31^{\circ} 11^{\prime} \mathrm{E}$; 4. Relt yarsh Lake, Vuim Mt., $68^{\circ} 06^{\prime} \mathrm{N} 31^{\circ} 18^{\prime} \mathrm{E}$; 5. Konicheskay Mt., $68^{\circ} 06^{\prime} \mathrm{N} 31^{\circ} 23^{\prime} \mathrm{E}$; 6. Elgoras

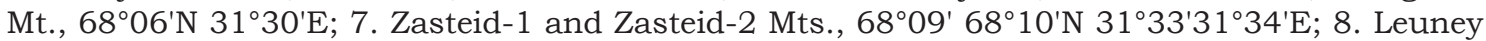
Mt. and Leuney Stream, $68^{\circ} 10^{\prime} \mathrm{N} 31^{\circ} 30^{\prime} \mathrm{E}$; 9. Vuva River Valley, $68^{\circ} 04^{\prime} \mathrm{N} 31^{\circ} 05^{\prime} \mathrm{E}$; Chuna-tundra Mts. (ChT): 10. El'nyun Mt. and El'yavr Lake, $67^{\circ} 39^{\prime} \mathrm{N} 32^{\circ} 36{ }^{\prime} \mathrm{E} ; 11$. Seydnotlag Place, $67^{\circ} 41^{\prime} \mathrm{N}$ $32^{\circ} 23^{\prime} \mathrm{E}$; 12 . Kupletskogo Stream, $67^{\circ} 42^{\prime} \mathrm{N} 32^{\circ} 23^{\prime} \mathrm{E}$; 13 . Namlagchorr and Ebruchorr Mts., $67^{\circ} 49^{\prime} \mathrm{N}$ $32^{\circ} 24^{\prime} \mathrm{E}$; 14 . middle part of the Suen laguay Stream, $67^{\circ} 51^{\prime} \mathrm{N} 32^{\circ} 22$ 'E; 15 . Lemkorr Canyon and Seydyavr Lake, $67^{\circ} 53^{\prime} \mathrm{N} 32^{\circ} 33^{\prime} \mathrm{E}$; Monche-tundra Mts. (MT): 16. Khipik Mt., 67 $57^{\circ} \mathrm{N} 32^{\circ} 32^{\prime} \mathrm{E} ; 17$. Vaykis Lake and Vaykisyavrlag Mt., $68^{\circ} 01^{\prime} \mathrm{N} 32^{\circ} 26^{\prime} \mathrm{E}$; 18 . Krasnaya Lambina Lake, $68^{\circ} 03^{\prime} \mathrm{N} 32^{\circ} 36^{\prime} \mathrm{E}$; Nyavka-tundra Mts. (NT): 19. Nizhniy Sylpyay Place, $68^{\circ} 02^{\prime} \mathrm{N} 32^{\circ} 40^{\prime} \mathrm{E} ; 20$. Verkhniy Sylpyay Place,

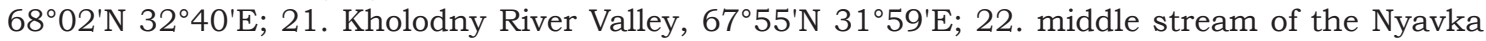

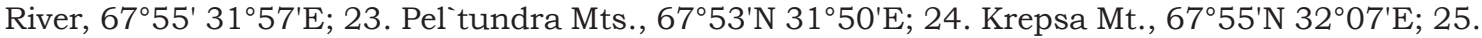
Mavra River Place, $67^{\circ} 41^{\prime} \mathrm{N} 31^{\circ} 51^{\prime} \mathrm{E}$.

Species included in the Red Data Book of Murmansk Province (Red Data Book..., 2014; Decree of Government of Murmansk Province ..., 2014) are marked as MP, in the Red Data Book of the
Russian Federation (2008) as RF, and in the Red Data Book of European Bryophytes (1995) as EB. When a taxon was not collected but is referred to in publications, the source is given. 
ANASTROPHYllum SPHENOLOBOIDES R.M. Schust. Unique. ST: 2 (Bakalin, 2004). EB: V (Vulnerable), MP: 2 (Vulnerable).

AnEURA PINGUis (L.) Dumort. - Not rare. ST: 1, 2, 3, 4, 7, 9; ChT: 10, 12, 14; MT: 17; NT: $21,22,23$.

AnTHELIA JUlacea (L.) Dumort. - Rare. ST: 7; ChT: 10; MT: 17.

ANTHELIA JURATZKANA (Limpr.) Trevis. - Frequent. ST: 1, 2, 3, 4, 5, 6, 7, 8; ChT: 10, 11, 12, 13, 14, 15; MT: 16, 17, 18; NT: 19, 20, 21, 22, 23, 24, 25.

ARNELLIA FENNICA (Gottsche) Lindb. - Unique. MT: 17; NT: 23. EB: R (Rare), MP: 3 (Rare).

BARBILOPHOZIA BARBATA (Schmidel ex Schreb.) Loeske-Sporadic. ST: 1, 3, 4, 7, 9; ChT: 10, 11, 12; MT: 16, 17, 18; NT: 21, 23.

BARBILOPHOZIA HATCHERI (A. Evans) Loeske - Not rare. ST: 1, 2, 4, 5, 6, 7, 8; ChT: 10, 11, 13, 14, 15; MT: 16, 17, 18; NT: 20, 21, 22, 23, $24,25$.

BARBILOPHOZIA LYCOPODIOIDES (Wallr.) Loeske [Lophozia lycopodioides (Wallr.) Cogn.] - Frequent. ST: 2, 3, 4, 6, 7, 8, 9; ChT: 10, 11, 12, 13, 14, 15; MT: 16, 17, 18; NT: 19, 20, $21,22,23,24,25$.

Barbilophozia Rubescens (R. M. Schust. \& Damsh.) Kartt. \& L. Soederstr. - Rare. NT: 20, 22. EB: K (insufficiently known), MP: 3 (Rare).

BLASIA PUSILLA L. - Sporadic. ST: 1, 4, 7, 8; ChT: 10, 12, 14; MT: 17; NT: 23.

BlePHAROSTOMA TRICHOPHYLLUM (L.) Dumort. var. TRICHOPHYLLUM - Frequent. ST: 1, 2, 3, 4, 5, 7, 8, 9; ChT: 10, 11, 12, 13, 14, 15; MT: 16, 17, 18; NT: 19, 20, 21, 22, 23, 24.

BLEPHAROSTOMA TRICHOPHYLLUM var. BREVIRETE Bryhn \& Kaal. - Rare. ChT: 13, 14; MT: 16, 17; NT: 24.

CALYPogeia InTEgristipula Steph. - Frequent. ST: 1, 2, 4, 7, 9; ChT: 10, 11, 13, 14; MT: 17, 18; NT: 21, 22, 23, 24.

Calypogeia muelleriana (Schiffn.) Müll. Frib. Sporadic. ST: 1, 3, 4, 6, 7, 9; ChT: 10, 14, 15; NT: 21, 23.

Calypogeia neesiana (C. Massal. \& Carestia) Müll. Frib. - Sporadic. ST: 1, 2, 3, 7, 9; ChT: 10, 11, 14; MT: 17; NT: 23.

Calypogeia sphagnicola (Arnell \& J. Perss.) Warnst. \& Loeske - Sporadic. ST: 1, 2, 8, 9; ChT: 10, 11, 14; MT: 17; NT: 23, 25.

Calypogeia suecica (Arnell \& J. Perss.) Müll. Frib. - Unique. ST: 7; NT: 21. MP: 3 (Rare).
Cephalozia bicuspidata (L.) Dumort. subsp. BicusPIDATA - Frequent. ST: 1, 2, 3, 4, 5, 6, 7, 8, 9; ChT: 10, 11, 12, 13, 14, 15; MT: 16, 17, 18; NT: 19, 20, 21, 22, 23, 24.

Cephalozia Bicuspidata subsp. ambigua (C. Massal.) R.M.Schust. - Sporadic. ST: 5, 7, 8; ChT: 12, 13, 14; MT: 17; NT: 21, 22.

Cephaloziella divaricata (Sm.) Schiffn. - Sporadic. ST: 1, 2, 9; ChT: 10, 12, 15; MT: 16, 17; NT: 19, 21.

Cephaloziella elachista (J. B. Jack ex Gottsche \& Rabenh.) Schiffn. - Rare. ST: 9; ChT: 12. EB: K (insufficiently known), MP: 3 (Rare).

Cephaloziella grimsulana (J. B. Jack ex Gottsche \& Rabenh.) Lacout. s. str. - Unique. ST: 4; ChT: 12.

Cephaloziella hampeana (Nees) Schiffn. - Rare. ST: 5, 9; ChT: 10.

Cephaloziella Rubella (Nees) Warnst. - Rare. ChT: 11, 12; MT: 18; NT: 21.

CEPHALOZIElla SPINIGERA (Lindb.) Warnst. [Cephaloziella subdentata Warnst.] - Sporadic. ST: 2; ChT: 10; MT: 17.

CePhaloziella VARIans (Gottsche) Steph. [Cephaloziella arctica Bryhn \& Douin] - Rare. ST: 2, 4; ChT: 13; MT: 16, 17.

Chiloscyphus pallescens (Ehrh. ex Hoffm.) Dumort. var. PALLESCENS - Unique. MT: 17; NT: 22.

Chiloscyphus polyanthos (L.) Corda - Sporadic. ST: 1, 3, 9; ChT: 11; MT: 16, 17, 18; NT: 21, 22, 23, 24, 25.

Clevea hyalina (Sommerf.) Lindb. [Athalamia hyalina (Sommerf.) S. Hatt.] - Unique. MT: 17; NT: 25. MP: 3 (Rare).

Conocephalum Conicum (L). Dumort. s. str. Unique. ST: 6; ChT: 12. MP: species requires special attention in Murmansk Province.

Conocephalum SAlEbrosum Szweyk., Buczk. \& Odrzyk. - Rare. ST: 1; ChT: 11, 12; NT: 21.

Crossocalyx hellerianus (Nees ex Lindenb.) Meyl. - Rare. ST: 1, 7; ChT: 11, 12; NT: 21, 23. MP: 3 (Rare).

Diplophyllum ALBICANS (L.) Dumort. - Sporadic. ST: 2, 4; ChT: 13, 14, 15; MT: 17; NT: 22.

Diplophyllum obTUSIFOlium (Hook.) Dumort. Rare. ST: 1, 2; MT: 17, 18.

Diplophyllum TAXIFOlIUM (Wahlenb.) Dumort. Not rare. ST: 1, 2, 3, 4, 5, 6, 7, 8; ChT: 10, 11, 13, 14; MT: 16, 17, 18; NT: 19, 20, 21, $22,23,24$.

Endogemma caespiticia (Lindenb.) Konstant., Vilnet \& Troitsky [Solenostoma caespiticium 
(Lindenb.) Steph.] - Rare. ST: 1, 2; MT: 16, 17.

EREMonotus MYRIOCARPUS (Carrington) Pearson - Unique. ST: 8. MP: 3 (Rare). MP: 3 (Rare).

Fossombronia IncuRva Lindb. - Unique. ChT: 10 (Andrejeva, 2009). EB: R (Rare), MP: species requires special attention in Murmansk Province.

Frullania tamarisci (L.) Dumort. - Unique. NT: 23. MP: 2 (Vulnerable).

Fuscocephaloziopsis ALBESCENS (Hook.) Váňa \& L.Söderstr. [Pleurocladula albescens (Hook.) Grolle] - Not rare. ST: 1, 2, 3, 4, 5, 7, 9; ChT: $11,13,14,15$; MT: 16,17 ; NT: 21 , 22, 23, 24.

Fuscocephaloziopsis CONNIVENs (Dicks.) Váňa \& L.Söderstr. [Cephalozia connivens (Dicks.) Lindb.] - Unique. ChT: 12; MT: 17. MP: species requires special attention in Murmansk Province.

Fuscocephaloziopsis leUCANTHA (Spruce) Váña \& L.Söderstr. [Cephalozia leucantha Spruce] Sporadic. ST: 1, 3, 6, 7, 9; ChT: 10, 11, 14, 15; MT: 17, 18; NT: 21, 23, 24.

FUSCOCEPHALOZIOPSIS LOITLESBERGERI (Schiffn.) Váňa \& L.Söderstr. [Cephalozia loitlesbergeri Schiffn] - Sporadic. ST: 1, 3, 7, 9; ChT: 10, 11; MT: 17; NT: 21.

FUSCOCEPHALOZIOPSIS LUNULIFOLIA (Dumort.) Váña \& L.Söderstr. [Cephalozia lunulifolia (Dumort.) Dumort.] - Not rare. ST: 1, 2, 4, 6, 7, 8, 9; ChT: 10, 12, 14; MT: 16, 17, 18; NT: 21, 22, 23, 24.

Fuscocephaloziopsis Pleniceps (Austin) Váňa $\&$ L.Söderstr. [Cephalozia pleniceps (Austin) Lindb.] - Sporadic. ST: 1, 3, 6, 7, 9; ChT: 10, 11, 14; NT: 23.

Geocalyx Graveolens (Schrad.) Nees - Sporadic. ST: 1, 3, 7; ChT: 10, 11; MT: 17, 18; NT: 21.

Gymnocolea Inflata (Huds.) Dumort. - Frequent. ST: 1, 2, 3, 4, 6, 7, 8, 9; ChT: 10, 11, 12, 13, 14, 15; MT: 16, 17, 18; NT: 19, 21, 22; 23, 24.

GYMNOMITRION BREVISSIMUM (Schleich. ex Dumort.) Warnst [Marsupella brevissima (Dumort.) Grolle] - Sporadic. ST: 2, 3, 4, 5, 6, 7; ChT: 10, 13, 14, 15; MT: 16.

GyMNOMITRION CONCINNATUM (Lightf.) Corda - Not rare. ST: 2, 3, 5, 6, 7, 8; ChT: 10, 11, 13, 14, 15; MT: 16, 17, 18; NT: 19, 20, 21, 22, 23, 24.

GYMNOMITRION CORALLIOIDES Nees - Sporadic. ST: 2, 4, 5, 6; ChT: 13, 14; MT: 16, 17; NT: 23, 24.
HAPLOMitrium HOOKERI (Sm.) Nees - Rare. ChT: 12; NT: 23, 25. RF: 2a (Vulnerable), MP: 3 (Rare). HARPANTHUS FLOTOVIANUS (Nees) Nees - Frequent. ST: 1, 2, 3, 4, 7, 8, 9; ChT: 10, 11, 12, 14; MT: 17, 18; NT: 21, 22, 23, 24, 25.

HETEROGEMma LAXA (Lindb.) Konstant. \& Vilnet [Schistochilopsis laxa (Lindb.) Konstant., Lophozia laxa (Lindb.) Grolle] - Unique. ST: 4; NT: 23. EB: RT (Regionally Threatened); MP: 3 (Rare).

Hygrobiella LaXIFOlia (Hook.) Spruce - Rare. ST: 1, 6; ChT: 13, 15; MT: 17.

IsOPACHES BICRENATUS (Schmidel ex Hoffm.) H. Buch [Lophozia bicrenata (Schmidel ex Hoffm.) Dumort.] - Frequent. ST: 1, 2, 3, 4, 5, 6, 7, 8, 9; ChT: 10, 11, 12, 13, 14, 15; MT: 16, 17, 18; NT: 19, 20, 21, 22, 23, 24; 25.

JungeRMANNIA BOREALIS Damsh. \& Váňa - Sporadic. ST: 2, 4, 6, 7; MT: 17; NT: 23, 24.

JUNGERMANNIA EUCORDIFOLIA Schljakov [Solenostoma cordifolium (Dumort.) Steph.] - Not rare. ST: 2, 4, 5, 7, 8; ChT: 10, 11, 12, 14; MT: 16, 17; NT: 22, 23, 24.

JUNGERMANNIA POLARIS Lindb. - Rare. ST: 4, 8; MT: 17.

JungeRMANNIA PUMILA With. - Unique. ST: 1; ChT: 15.

KURZIA PAUCIFloRa (Dicks.) Grolle - Unique. ST: 9; ChT: 12. MP: 3 (Rare).

LEJEUNEA CAVIFOLIA (Ehrh.) Lindb. - Unique. ST: 4; MT: 17. MP: 2 (Vulnerable).

LEPIDOZIA REPTANS (L.) Dumort. - Sporadic. ST: 1, 2, 7; ChT: 10, 15; MT: 17; NT: 20, 21.

LiOCHLAENA LANCEOLATA Nees [Jungermannia leiantha Grolle] - Sporadic. ST: 8; ChT: 10, 12; MT: 17, 18; NT: 21.

LOPHOCOLEA HETEROPHYLla (Schrad.) Dumort. Sporadic. ST: 1, 7; ChT: 10, 11, 12; NT: 20, 21; MT: 17.

LOPHOCOLEA MINOR Nees - Rare. ST: 7, 8; ChT: 12; MT: 17.

LOPHOZIA ASCENDENS (Warnst.) R. M. Schust. Unique. ST: 7; NT: 21. EB: R (Rare), MP: 3 (Rare).

LoPHOZIA GUTTULATA (Lindb. \& Arnell) A. Evans Not rare. ST: 1, 2, 3, 4, 8, 9; ChT: 10, 11, 12; MT: 16, 17, 18; NT: 19, 20, 21, 22.

LOPHOZIA SAVICZIAE Schljakov - Rare. ST: 1, 2; ChT: 15; NT: 24.

LOPHOZIA SCHUSTERIANA Schljakov - Unique. ChT: 15 (Bakalin, 2005).

LOPHOZIA SILVICOLA H. Buch - Not rare. ST: 1, 2, 7 , 8; ChT: 10, 12, 13, 15; MT: 16, 17; NT: 21, 23. 
LOPHOZIA VENTRICOSA var. LONGIFLORA (Nees) Macoun - Not rare. ST: $1,2,3,4,6,7,8$; ChT: 10, 12, 14, 15; MT: 16, 17, 18; NT: 21, 22, 23, 24.

LOPHOZIA WENZELII var. GROENLANDICA (Nees) Bakalin - Not rare. ST: 2, 4, 5, 7, 8, 9; ChT: 10, 12, 13, 15; MT: 16, 17; NT: 20, 21, 22, 23, 24.

LOPHOZIA WENZELII var. LAPPONICA H. Buch $\& \mathrm{~S}$. W. Arnell - Rare. ST: 1; MT: 17; NT: 24.

LOPHOZIA WENZELII var. LITORALIS (Arnell) Bakalin - Unique. ST: 3; ChT: 15.

LOPHOZIA WENZELII (Nees) Steph. var. WENZELII Sporadic. ST: 1, 7, 8; ChT: 10, 12, 13, 15; MT: 16, 17, 18; NT: 21, 25.

LoPHOZIOPSIS EXCISA (Dicks.) Konstant. \& Vilnet var. EXCISA [Lophozia excisa (Dicks.) Dumort. var. excisa] - Sporadic. ChT: 12; MT: 16, 17, 18; NT: 19, 20, 21, 22, 25.

LOPHOZIOPSIS EXCISA var. ELEGANS (R. M. Schust.) Konstant. \& Vilnet [Lophozia excisa var. elegans R.M. Schust.] - Unique. MT: 17.

LOPHOZIOPSIS LONGIDENS (Lindb.) Konstant. \& Vilnet [Lophozia longidens (Lindb.) Macoun] - Not rare. ST: 1, 2, 3, 6, 7, 8; ChT: 10, 11, 12, 15; MT: 16, 17, 18; NT: 19, 20, 21, 25.

LOPHOZIOPSIS PROPAGULIFERA (Gottsche) Konstant. \& Vilnet [Lophozia propagulifera (Gottsche) Steph.] - Rare. ChT: 10, 14; MT: 17.

MANNIA GRACILIS (F. Weber) Schill \& D.G. Long [Asterella gracilis (F. Weber) Underw.] - Rare. ST: 7; NT: 23, 24.

Mannia PILOSA (Hornem.) Frye \& L. Clark Unique. MT: 7. MP: 2 (Vulnerable).

MANNIA TRIANDRA (Scop.) Grolle - Unique. ST: 7. EB: R (Rare).

MARCHANTIA POLYMORPHA L. subsp. POLYMORPHA [M. aquatica (Nees) Burgeff]. Rare. ST: 4, 8; ChT: 11; NT: 21, 22.

MARCHANTIA POLYMORPHA subsp. MONTIVAGANS Bischl. \& Boissel.-Dub. [M. alpestris (Nees) Burgeff.] - Sporadic. ST : 2, 3, 8 ; ChT: 10, 11 ; MT: 16, 17; NT: 21, 22, 23, 24.

MARCHANTIA POLYMORPHA subsp. RUDERALIS Bischl. $\&$ Boissel.-Dub. [M. latifolia Gray] - Frequent. ST: 1, 2, 3, 4, 6, 7, 9; ChT: 10, 11, 12, 14; MT: 16, 17, 18; NT: 19, 20, 21, 22, 23, 24, 25.

Marsupella apiculata Schiffn. - Sporadic. ST: 2, 4, 5, 7, 9; ChT: 11, 13, 14; MT: 16, 17; NT: 23, 24.

Marsupella aquatica (Lindenb.) Schiffn. - Sporadic. ST: 2, 4, 5, 6, 7, 8; ChT: 10, 13, 14; MT: 16, 17; NT: 23, 24.
Marsupella boeckil (Austin) Kaal. - Not rare. ST: 2, 4, 5, 7, 8; ChT: 13, 14, 15; MT: 16, 17; NT: 24.

Marsupella condensata (Ångstr. ex C.Hartm.) Kaal. - Sporadic. ST: 4, 5, 6, 8; ChT: 13, 15; MT: 16, 17.

Marsupella EMARginata (Ehrh.) Dumort. - Sporadic. ST: 5, 6, 7, 8; ChT: 10, 12, 14; MT: $16,17$.

MarSuPELla SPHACELATA (Giesecke ex Lindenb.) Dumort. - Unique. ST: 8; MT: 17.

MARSUPElla SPRUCEI (Limpr.) Bernet - Rare. ST: 4, 6, 7; ChT: 13; MT: 16.

MEsoptychia BADENSIS (Gottsche ex Rabenh.) L.Söderstr. \& Váňa [Leiocolea badensis (Gottsche) Jørg.] - Unique. MT: 17. MP: 3 (Rare).

MesoptyChia GILLMANII (Austin) L.Söderstr. \& Váňa [Leiocolea gillmanii (Austin) A. Evans] - Sporadic. ST: 5, 6, 7; ChT: 10, 11, 12, 15; MT: 17; NT: 23, 24.

Mesoptychia heterocolpos (Thed. ex Hartm.) L.Söderstr. \& Váňa [Leiocolea heterocolpos (Thed. ex Hartm.) H. Buch] - Sporadic. ST: 4, 5, 6, 7; ChT: 11, 12, 13; MT: 17; NT: 23, 24.

MEsoptYCHIA RUTHEANA (Limpr.) L.Söderstr. \& Váňa [Leiocolea rutheana (Limpr.) Müll.Frib.] Rare. ChT: 13; MT: 17.

MetZGeria FURCATA (L.) Dumort. - Rare. ST: 4, 6; ChT: 12; MT: 16. MP: 3 (Rare).

MoERCKIA BLYTTII (Moerck in Hornem.) Brockm.Sporadic. ST: 4, 6, 8; ChT: 11, 13; MT: 17.

MoERCKIA Flotoviana (Nees) Schiffn. [M. hibernica (Hook.) Gottsche f. flotoviana] - Sporadic. ST: 3, 8; ChT: 12; MT: 17; NT: 23.

Mylia ANomala (Hook.) Gray - Frequent. ST: 1, 2, 3, 5, 6, 7, 9; ChT: 10, 11, 12, 14; MT: 16, 17, 18; NT: 19, 20, 21, 22, 23, 24.

MYLIA TAYLORI (Hook.) Gray - Rare. ST: 4, 6, 7; ChT: 11; MT: 17.

NARDIA BREIDLERI (Limpr.) Lindb. - Rare. ST: 1, 7; ChT: 15. RF: 4 (insufficiently known); MP: 5 (special status).

NARDiA GEOScyphus (De Not.) Lindb. - Not rare. ST: 2, 6, 7, 8, 9; ChT: 10, 12, 13, 15; MT: 16, 17, 18; NT: 19, 20, 23, 24.

NARDIA INSECTA Lindb. - Sporadic. ST: 1, 2, 3, 4, 5, 6, 7; ChT: 10, 12, 13, 15; NT: 21.

NARDIA JAPONICA Steph. - Rare. ST: 1; ChT: 12, 15; NT: 23.

NARDIA SCALARIS Gray - Sporadic. ST: 4, 5, 6, 7; ChT: 13, 15; MT: 16; NT: 20, 24.

NEOORTHOCAulis atTEnUatus (Mart.) L. Söderstr., De Roo \& Hedd. [Orthocaulis attenuatus 
(Mart.) A. Evans, Barbilophozia gracilis Müll. Frib.] - Sporadic. ST: 1, 2, 3, 7, 9; ChT: 10, 11, 14; MT: 16, 17, 18.

NeOORTHOCAulis BINSTEADII (Kaal.) L. Söderstr., De Roo \& Hedd. [Orthocaulis binsteadii (Kaal.) H.Buch, Barbilophozia binsteadii (Kaal.) Loeske] - Sporadic. ST: 1, 2, 4, 6, 7; 8, 9; ChT: 10, 12, 14; MT: 16, 17, 18; NT: 20, 21, 22, 23, 24.

NeOoRThocaulis floerkei (F. Weber \& D. Mohr) L. Söderstr., De Roo \& Hedd. [Orthocaulis floerkei (F. Weber \& D. Mohr) H. Buch, Barbilophozia floerkei (F. Weber \& D. Mohr) Loeske] - Not rare. ST: 1, 2, 6, 7, 8, 9; ChT: 10, 11, 14; MT: 17, 18; NT: 21, 22, 23, 24.

Nowellia CURVIFolia (Dicks.) Mitt. - Unique. ST: 7. MP: species requires special attention in Murmansk Province.

Obtusifolium obTUsum (Lindb.) S.W. Arnell - Sporadic. ST: 1, 2, 4, 7, 9; ChT: 10, 11, 12; 14; MT: 16, 17, 18; NT: 21, 22.

Odontoschisma Elongatum (Lindb.) A. Evans Not rare. ST: 1, 2, 3, 4, 6, 7, 8, 9; ChT: 10, 11, 13, 15; MT: 16, 17; NT: 21, 22, 23, 24.

Odontoschisma Fluitans (Nees) L.Söderstr. \& Vána [Cladopodiella fluitans (Nees) $\mathrm{H}$. Buch] - Sporadic. ST: 1, 2, 3, 4, 5, 7, 8, 9; ChT: 10, 11, 14; MT: 17, 18; NT: 21, 22, 23, 24.

ODONTOSCHISMA FRANCISCI (Hook.) L.Söderstr. \& Váňa [Cladopodiella francisci (Hook.) Jørg.] Rare. ST: 1, 5, 9; ChT: 15; MT: 18.

OdONTOSCHISMA MACOUNII (Austin) Underw. - Rare. ST: 7; ChT: 11, 12; MT: 17; NT: 23.

Oleolophozia PERssoniI (H. Buch \& S. W. Arnell) L. Söderstr., De Roo \& Hedd. [Lophoziopsis perssonii (H.Buch \& S.W.Arnell) Konstant. \& Vilnet, Lophozia perssonii H. Buch \& S.W. Arnell] - Unique. MT: 17. RF: 3 (Rare), MP: 2 (Vulnerable).

Pellia EPIPhylla (L.) Corda - Sporadic. ST: 1, 2, 4, 6, 7; ChT: 10, 14; NT: 21, 22, 23.

PELlia NEESIANA (Gottsche) Limpr. - Not rare. ST: 1, 2, 7, 8; ChT: 10, 11, 12, 13, 15; MT: 17, 18; NT: 20, 21, 22, 23, 24.

Peltolepis QUadRata (Saut.) Müll. Frib. - Unique. ST: 7; MT: 17. MP: 3 (Rare).

Plagiochila PORELloIDES (Torrey ex Nees) Lindenb. - Sporadic. ST: 1, 3, 7; ChT: 11, 12; MT: 17; NT: 20, 21.

Plectocolea hyalina (Lyell) Mitt. - Sporadic. ST: 3, 7, 9; ChT: 10, 13, 14; MT: 17; NT: 24.

Plectocolea obovata (Nees) Lindb. - Sporadic. ST: 2, 4, 5, 6, 7; ChT: 10, 13, 15; MT: 17.
Plectocolea subelliptica (Lindb. ex Kaal.) A. Evans - Sporadic. ST: 6, 7; ChT: 13, 14; NT: 22, 24.

Porella CORdaEANa (Huebener) Moore - Unique. NT: 21. MP: species requires special attention in Murmansk Province.

Porella Platyphylla (L.) Pfeiff. - Unique. MT: 17. MP: 3 (Rare).

PRASANTHUS SuEcicus (Gottsche) Lindb. - Unique. ChT: 13; MT: 17. MP: 3 (Rare).

PREISSIA QUADRATA (Scop.) Nees - Sporadic. ST: 2, 4, 5, 6, 7; ChT: 10, 12, 13, 14; MT: 16, 17; NT: 23, 24.

Protolophozia elongata (Steph.) Schljakov [Lophozia elongata Steph.] - Unique. ChT: 15; MT: 17. EB: K (insufficiently known), RF: 2a (Vulnerable), MP: 3 (Rare).

Pseudolophozia DeBiliformis (R.M.Schust. \& Damsh.) Konstant. \& Vilnet var. DEBILIFORMIS [Protolophozia debiliformis (R.M.Schust.) Konstant. var. debiliformis] - Unique. ST: 4, 5 .

PSEUdOLOPHOZIA DEBILIFORMIS var. CONCOLOR (R.M.Schust.) Konstant. \& Vilnet [Lophozia debiliformis var. concolor R.M.Schust. \& Damsh.] - Unique. ChT: 15 (Konstantinova, Borovichev, 2006).

Pseudolophozia sudetica (Nees ex Huebener) Konstant. \& Vilnet var. SUDETICA [Lophozia sudetica (Nees ex Huebener) Grolle] - Frequent. ST: 1, 2, 3, 4, 5, 6, 7; ChT: 10, 11 , 12, 13, 14, 15; MT: 16, 17; NT: 21, 23, 24.

PSEUdOLOPHOZIA SUdETICA var. ANOMALA (Schljakov) Konstant. \& Vilnet [Lophozia sudetica (Nees ex Hueb.) Grolle var. anomala (Schljakov) Schljakov] - Unique. ST: 2; ChT: 15.

Ptilidium Ciliare (L.) Hampe - Frequent. ST: 1, 2, 4, 5, 6, 7, 8; ChT: 10, 11, 13, 14; MT: 16, 17, 18; NT: 19, 20, 21, 22, 23, 24; 25.

PTILIDIUM PULCHERRIMUM (Weber) Vain. - Frequent. ST: 1, 2, 3, 7, 9; ChT: 10, 11, 12; MT: 16, 17, 18; NT: 19, 20, 21, 23, 24, 25.

Radula COMPlanata (L.) Dumort. - Sporadic. ST: 6, 7, 8; ChT: 11; NT: 25; MT: 17, 18. MP: species requires special attention in Murmansk Province.

Radula lindBergiana Gottsche ex C. Hartm. Unique. ChT: 11; MT: 17.

Reboulia HEMisphaerica (L.) Raddi - Unique. ST: 7. MP: species requires special attention in Murmansk Province.

RICCARDIA CHAMEDRYFOLIA (With.) Grolle - Rare. ST: 1, 2, 9; MT: 18. 
RICCARDIA LATIFRONS (Lindb.) Lindb. - Sporadic. ST: 1, 3, 4, 6, 8, 9; ChT: 15; MT: 17; NT: 21,23

RicCARDIA MULTIFIDA (L.) Gray - Unique. NT: 25 (Andrejeva, 2005). MP: species requires special attention in Murmansk Province.

RICCARDIA PALMATA (Hedw.) Carruth. - Unique. ST: 1; NT: 21. MP: 3 (Rare).

SACCOBASIS POLITA (Nees) H. Buch - Sporadic. ST: 4, 6, 7, 8, 9; ChT: 10, 11, 13, 14; MT: 16, 17, 18; NT: 21, 22, 23, 24.

SACCOBASIS POLYMORPHA (Schust.) Schljakov Rare. ChT: 10, 15.

SAUTERIA ALPINA (Nees) Nees - Unique. ST: 7. MP: 3 (Rare).

SCAPANIA APICULATA Spruce - Unique. ST: 1. MP: 3 (Rare).

SCAPANIA CALCICOLA (Arnell \& J.Perss.) Ingham Unique. MT: 17. MP: species requires special attention in Murmansk Province.

SCAPANIA CRASSIRETIS Bryhn - Unique. ChT: 15; MT: 17.

SCAPANIA CURTA (Mart.) Dumort. - Sporadic. ST: 1, 2, 3, 4, 5, 6, 7; ChT: 10, 13, 14; MT: 17; NT: 21.

SCAPANia CUSPiduligera (Nees) Müll.Frib. Unique. MT: 17; NT: 24.

SCAPANIA GYMNOSTOMOPHILA Kaal. - Unique. ChT: 11; MT: 17.

SCAPANIA HYPERBOREA Jørg. - Sporadic. ST: 1, 2, 3 , 4, 5, 6, 7, 8, 9; ChT: 10, 11, 13; NT: 23, 24.

SCAPANIA IRRIGUA (Nees) Nees - Not rare. ST: 1,2 , 3, 4, 5, 6, 7, 8, 9; ChT: 10, 11, 12, 14; MT: 16, 17, 18; NT: 19, 20, 21, 22, 23, 24

SCAPANIA KAURINII Ryan - Unique. NT: 24. MP: 3 (Rare).

ScAPANIA Lingulata H. Buch - Unique. ChT: 15 (Konstantinova, Borovichev, 2006)

SCAPANIA MUCRONATA H. Buch - Sporadic. ST: 1, 2, 3, 4, 6, 7; ChT: 10, 11, 14; MT: 18; NT: 21.

SCAPANIA OBCORDATA (Berggr.) S. W. Arnell - Rare. ST: 5, 6, 7; NT: 24 .

SCAPANIA OBSCURA (Arnell \& C. E. O.Jensen) Schiffn. - Unique. ST: 4 (Bakalin, 2004). MP: species requires special attention in Murmansk Province.

SCAPANia PALUdicola Loeske \& Müll.Frib. - Sporadic. ST: 1, 3, 5, 6, 7, 9; ChT: 10, 11, 14; MT: 16, 17; NT: 21, 22, 23, 24.

ScAPANia Paludosa (Müll.Frib.) Müll.Frib. - Sporadic. ST: 1, 3, 4, 5, 6, 7, 8, 9; ChT: 10, 13; MT: 17, 18.
SCAPANIA PARVIFOLIA Warnst. - Sporadic. ST: 1, 5, 8; ChT: 10, 13; MT: 17, 18. EB: T (taxa apparently threatened but presenting taxonomic problems).

SCAPANIA PRAETERVISA Meyl. - Sporadic. ChT: 10, 13, 14; MT: 17; NT: 21, 24.

SCAPANIA SCANDICA (Arnell \& H.Buch) Macvicar Sporadic. ST: 1, 2, 5, 6, 7; ChT: 10, 13, 14; MT: 16, 17; NT: 19, 20, 21, 23.

SCAPANIA SIMMONSII Bryhn \& Kaal. - Unique. ST: 7. EB: R (Rare), MP: 2 (Vulnerable).

SCAPANiA SPITSBERGENSIS (Lindb.) Müll.Frib. Unique. MT: 17. EB: R (Rare), MP: 3 (Rare).

SCAPANIA SUBALPINA (Nees ex Lindenb.) Dumort. - Frequent. ST: 2, 3, 4, 5, 6, 7, 8; ChT: 10, $11,12,13,14,15$; MT: 16, 17; NT: 20, 21, 22, 23, 24, 25.

SCAPANIA TUNDRAE (Arnell) H. Buch - Unique. MT: 17. EB: R (Rare), MP: 3 (Rare).

ScAPANia Uliginosa (Sw. ex Lindenb.) Dumort. Sporadic. ST: 2, 5, 7, 9; ChT: 10, 11, 13,14; MT: 17; NT: 23.

Scapania umbrosa (Schrad.) Dumort. - Unique. NT: 21. MP: 3 (Rare).

SCAPANiA UNDUlata (L.) Dumort. - Not rare. ST: 1, 2, 3, 4, 5, 6, 7; ChT: 10, 11, 12, 13, 14; MT: 16, 17; NT: 21, 22, 23, 24.

SCHISTOCHILOPSIS GRANDIRETIS (Lindb. ex Kaal.) Konstant. - Rare. ST: 7, 8; MT: 17; NT: 23.

Schistochilopsis INCISA (Schrad.) Konstant. - Not rare. ST: 1, 2, 3, 4, 6, 7, 8, 9; ChT: 10, 11, 14; MT: 18; NT: 21.

SCHISTOCHILOPSIS OPACIFOLIA (Culm. ex Meyl.) Konstant. - Rare. ST: 5, 6, 8; ChT: 13; MT: 17.

SchLJAKOVIA KUNZEANA (Huebener) Konstant. \& Vilnet [Orthocaulis kunzeanus (Huebener) H. Buch] - Frequent. ST: 1, 2, 4, 5, 6, 7, 9; ChT: $10,11,12,13,14,15$; MT: 16, 17, 18; NT: 20, 21, 22, 23, 24.

SCHLJAKOVIANTHUS QUADRILOBUS (Lindb.) Konstant. $\&$ Vilnet [Orthocaulis quadrilobus (Lindb.) H. Buch] - Sporadic. NT: 4; ChT: 10, 13, 15; NT: 23, 24.

Solenostoma Confertissimum (Nees) Schljakov [Jungermannia confertissima Nees] - Sporadic. ST: 1, 7, 9; ChT: 10, 15; MT: 17; NT: 21, 23.

SolenOSTOMA SPHAEROCARPUM (Hook.) Steph. - Not rare. ST: 1, 2, 7, 9; ChT: 10, 13, 15; MT: 16, 18; NT: 21, 22, 24

Sphenolobus minutus (Schreb.) Berggr. [Anastrophyllum minutum (Schreb.) R. M. Schust.] 
- Frequent. ST: 1, 2, 4, 6, 7, 8, 9; ChT: 10, 11, 12, 13, 14; MT: 16, 17, 18; NT: 19, 20, 21, 22, 23, 24.

Sphenolobus SaXicola (Schrad.) Steph. - Sporadic. ST: 2, 5, 6, 7, 8; ChT: 10, 12, 13, 14; MT: 16, 17; NT: 20, 23, 24.

TETRALOPHOZIA SETIFORMis (Ehrh.) Schljakov [Chandonanthus setiformis (Ehrh.) Lindb.] - Frequent. ST: 1, 2, 4, 5, 6, 7, 8; ChT: 10, 11, 12, 13, 14, 15; MT: 16, 17; NT: 19, 20, 21, 22, 23, 24, 25.

TRITOMARIA EXSECTIFORMIS (Breidl.) Loeske Unique. MT: 18; NT: 21. MP: 3 (Rare).

TRitomaria Quinquedentata (Huds.) H.Buch Frequent. ST: 1, 2, 4, 5, 6, 7, 8; ChT: 10, 11, 13, 14, 15; MT: 16, 17; NT: 20, 21, 22, 23, 24, 25.

TRITOMARIA SCITUla (Taylor) Jørg. - Unique. ST: 5; ChT: 11.

\section{Excluded species}

CAlypogeia tRichomanis (L.) Corda - The record from Chuna-Tundra Mts. (Pushkina, 1960) seems to be erroneous. The voucher specimen was not found.

Cephaloziella uncinata R.M. Schust. - The species was cited for LSNBR by Bakalin (2004), but all specimens from LSNBR were reidentified as C. rubella (Yu.S. Mamontov, pers. com.).

Chiloscyphus FRAgilis (Roth) Schiffn. - The species was cited for LSNBR by Pushkina (1960) and Bakalin (2004), but the specimens were reidentified as Chiloscyphus pallescens var. pallescens.

HaRpanthus scutatus (F. Weber \& D. Mohr) Spruce - The record for mires of Mavra River (Pushkina, 1960) seems to be erroneous. The voucher specimen was not found.

MoERCKIA HIBERNICA (Hook.) Gottsche - All specimens of the species from LSNBR were reidentified as M. flotoviana (Yu.S. Mamontov, pers. com.).

FUSCOCEPHALOZIOPSIS ALBESCENS var. ISLANDICA (Nees) Váňa \& L.Söderstr. [Pleurocladula albescens var. islandica (Nees) Spruce] - The taxon was cited for LSNBR by Pushkina (1960), but the specimen (KPABG 31.VIII.1939) was reidentified as typical $F$. albescens.

ORTHOCAulis atlanticus (Kaal.) H. Buch - All records from LSNBR are erroneous.

\section{DISCUSSION}

As a result of the exploration 173 species (including 5 subspecies and 15 varieties) have been revealed. They belong to 3 classes - Haplomitriopsida (1 species), Marchantiopsida (12 species) and Jungermanniopsida (158 species). Nowadays, the liverwort flora of the Reserve comprises $63 \%$ of species in the liverwort flora of the European part of Russia, about $66,5 \%$ of those of north-west of Russia, and $88 \%$ of those of Murmansk Province. This means that the liverwort flora is the richest amongst floras of large State Nature Reserves of Russia.

In the course of our exploration, 52 hepatic species were reported for the first time for LSNBR. Among them eight liverworts are known in Murmansk Province only from the Reserve: Frullania tamarisci, Nowellia curvifolia (Borovichev, 2009), Lejeunea cavifolia (Konstantinova, Borovichev, 2006), Lophoziopsis excisa var. elegans (Borovichev, 2011), Mannia triandra, Reboulia hemisphaerica (Borovichev, Andrejeva, 2009), Oleolophozia perssonii (Borovichev, 2011) and Scapania calcicola (Borovichev, 2014).

Detailed studies of the liverwort flora of the LSNBR Mountains have revealed that the liverwort diversity varies in different areas: in Salnye Tundry Mts there were reported 139 species, in Monche-tundra Mts - 124, in Chuna-tundra Mts - 133. The lowest number was in Nyavka-tundra (112). 139 liverwort species have been found in the Salnye Tundry Mts, with 10 specific species for this area: these species grow exclusively on calcareous substrates (Mannia triandra, Reboulia hemisphaerica, Sauteria alpina) among them are arctic-montane species (Eremonotus myriocarpus, Scapania obscura), montane (Marsupella sphacelata), nemoral (Nowellia curvifolia, Scapania apiculata) and arctic species (Anastrophyllum sphenoloboides, Scapania simmonsii), with 4 species known only on this area (Borovichev, 2010). 133 liverwort species have been found in the Chuna-tundra Mts, and 6 species are specific for this place. Among them there are arctic species (Lophozia schusteriana), arctic-montane (Prasanthus suecicus, Saccobasis polymorpha), montane (Scapania lingulata) and boreal species (Fossombronia incurva, Riccardia multifida), with single species known in this area only. 124 species have been found in the Monche-tundra Mts, and six species are specific ones: arctic-montane species (Mesoptychia badensis, Oleolophozia 
perssonii, Mannia pilosa, Scapania spitsbergensis), arctic (Scapania tundrae) and nemoral (Porella platyphylla), with two taxa known only in this area (Borovichev, 2011). 112 species have been found in the Nyavka-tundra Mts, with five specific species: nemoral (Frullania tamarisci, Scapania umbrosa), arctic (Barbilophozia rubescens), montane (Porella cordeana), and arcticmontane species (Scapania kaurinit), with single species known in this area only (Borovichev, 2013). For all the examined liverwort floras of the LSNBR there were found 80 common species. Most of them are fairly abundant in the Murmansk Province. The share of these species in local floras varies between 58\% (Salnye Tundry Mts.) and 74.1\% (Nyavka-tundra Mts) depending on the flora's general species diversity. A fairly large number of species (30) has been noted for more than one flora, which is due to irregularity of previous studies of the LSNBR.

The high diversity of local liverwort floras can be accounted for by the variety of geological, geomorphological and micro-climatic conditions of the area, as well as by long exploration and fairly uniform coverage of the area by hepaticological studies. Further research will focus on more careful studying of hard-to-reach areas of the LSNBR.

In conclusion are offered a few words about the importance of conserving the hepatic flora of the LSNBR. Among the hepatics collected here, fifteen species are red-listed in Europe. One of them, Anastrophyllum sphenoloboides is vulnerable in Europe, nine (Arnellia fennica, Haplomitrium hookeri, Lophozia ascendens, Mannia triandra, Nardia japonica, Scapania spitsbergensis, S. simmonsii, S. tundrae) are rare species, four (Barbilophozia rubescens, Cephaloziella elachista, Pseudolophozia debiliformis, Protolophozia elongata) are insufficiently known and one (Scapania parvifolia) has an undetermined status. Four livervorts are included in the Red Data Book of the Russian Federation (2008) Haplomitrium hookeri, Nardia breidleri, Protolophozia elongata, Oleolophozia perssonii. Thirty one species are protected in the Region and ten taxa are species requiring special attention to their status in natural habitats of Murmansk Province (Red Data Book..., 2014; Decree of Government of Murmansk Province..., 2014). Therefore, the territory is quite representative for protection and sustainable development of rare hepatic species.

\section{ACNOWLEDGEMENTS}

I am very grateful to Prof. N. Konstantinova for confirmation of specimen identifications and constant encouragement. The administration of the Lapland State Nature Biosphere Reserve and Dr. A. Melekhin are thanked for their comprehensive assistance with organization of the field trips. Mr. A. Savchenko is thanked for preparing of maps. Thanks are also to Dr. Yu. Mamontov for revising of Cephaloziella and Moerckia specimens as well as Dr. N. Koroleva and Dr. N. Nikolaeva for improving the English of the manuscript and valuable comments. The work was partly supported by the Russian Foundation of Basic Research (grants 12-04-01476, 14-04-98810).

\section{REFERENCES}

Ahti T., Hämet-Ahti, L., Jalas, J. 1968. Vegetation zones and their sections in NorthernWestern Europe. Ann. Bot. Fennici 5: 169-211.

Andrejeva, E.N. 1990. Air pollution impact on the moss cover of the northern taiga. In: Forest ecosystems and air pollution. Leningrad, pp. 159-172. (In Russian).

Andrejeva, E.N. 2005. Structure of the moss cover under air pollution conditions. In: Ecological problems of plant communities. Sankt-Petersburg, pp. 105-129. (In Russian).

Andrejeva, E.N. 2009. New rare liverwort records from Russian Federation Regions. Arctoa 18: 281-286. (In Russian).

Bakalin, V.A. 2004. Liverworts of Lapland State Nature Reserve. In: Konstantinova (ed.) Liverworts and hornworts of the Russian State Nature Reserve: Modern state of biodiversity on reserved areas of Russia. Vol. 3. Lichens and Bryophytes. Moscow, pp. 236-273. (In Russian).

Bakalin, V.A. 2005. The monographic study of Lophozia (Dumort.) Dumort. s. str. Moscow, pp. 1-238. (In Russian).

Borovichev, E.A. 2009. Addition to liverworts flora of Lapland Reserve (Murmansk Region). Novosti Sist. Nizsh. Rast. 43: 313-321. (In Russian).

Borovichev, E.A. 2010. Liverworts (Marchanthiophyta) of Salnye Tundry Mts. (Lapland State Reserve, Murmansk Province). Novosti Sist. Nizsh. Rast. 44: 272-297. (In Russian).

Borovichev, E.A. 2011. To the liverworts flora (Marchantiophyta) of the Monche-tundra Mts. (Lapland State Reserve, Murmansk Region). Novosti Sist. Nizsh. Rast. 45: 276-291. (In Russian).

Borovichev, E.A. 2013. Liverworts flora (Marchantiophyta) of the Nyvka-Tundra Mts. (Lapland Nature State Reserve (Murmansk Region). Trudy Karel'skogo nauchnogo tsentra Ros. Akad. Nauk. 
Seriya Biogeografiya. 14(2): 46-54. (In Russian).

Borovichev, E.A. 2014. Scapania calcicola (Arnell \& J.Perss) Ingham - the new species for liverwort flora of Murmansk Province. Bjul. MOIP. Otd. Biol. 119 (2): 81-83. (In Russian).

Borovichev, E.A. \& Andrejeva, E.N. 2009. New liverwort records from Murmansk Province. 2. Arctoa 18: 249-250. (In Russian).

Decree of Government of Murmansk Province 25.04.2014 221-ПП/7 "About changes in decree of Government of Murmansk Province 04.09.2002 325-ПП" (including "List of Fungi, Lichens, Plants and Animals species included into the Red Data Book of Murmansk Province") (In Russian). http: / / www.pinro.ru/n22 /images / stories / news / 2014/redbook/2red.pdf

Heikinheimo, O. \& Raatikainen M. 1971. Paikan ilmoittaminen Suomesta talletetuissa biologisissa aineistoissa Ann. Entomol. Fenn. 37(1a): 1-27.

Konstantinova N.A. 2001. Hepatics in Strict Nature Reserves of European part of Russia Novit. Bot. Univ. Carol. 15: 77-93.

Konstantinova, N.A., Borovichev, E.A. 2006. To the liverwort (Hepaticae) flora of Murmansk Province Bot. Zhurn. 91(2): 322-328. (In Russian).

Konstantinova, N.A., Bakalin, V.A., Andreeva, E.N., Bezgodov, A.G., Borovichev, E.A., Dulin, M.V. \& Mamontov Yu.S. 2009. Checklist of liverworts (Marchantiophyta) of Russia. Arctoa 18: 1-63.

Pushkina, N.M. 1960. Lichens and bryophytes of the Lapland State Nature Reserve. In: Proceedings of the Lapland State Nature Reserve. Vol. 4. Moscow, pp. 189-248. (In Russian).

Red Data Book of European Bryophytes. 1995. Trondheim, 290 pp.

Red Data Book of Murmansk Province. 2014. Murmansk. (in press.). (In Russian).

Red Data Book of Russian Federation (plants and fungi). Moscow, 855 pp. (in Russian).

Rubasinghe, S.C.K., Long, D.G. \& Milne, R. 2011a. A new combination and three new synonyms in the genus Clevea Lindb. (Marchantiopsida, Cleveaceae). Journal of Bryology 33(2): 168-169. http:// dx.doi.org/ 10.1179/1743282011Y.0000000005
Rubasinghe, S.C.K., Milne, R., Forrest, L.L. \& Long, D.G. 2011b. Realignment of the genera of Cleveaceae (Marchantiopsida, Marchantiidae ). Bryologist 114: 116-127. http://dx.doi. org/10.1639/0007-2745-114.1.116

Schill, D.B., Long, D.G. \& Forrest L.L. 2010. A molecular phylogenetic study of Mannia (Marchantiophyta, Aytoniaceae) using chloroplast and nuclear markers. Bryologist 113(1): 164-179. http:// dx.doi.org/10.1639/0007-2745-113.1.164

Semyonov-Tyan-Schanskiy, O.I. 1988. Lapland State Nature Reserve. In: State Nature Reserves of USSR. State Nature Reserves of the European part of RSFSR. Part I. Moscow, p. 61-89. (In Russian).

Söderström, L., De Roo \& R., Hedderson, T. 2010. Taxonomic novelties resulting from recent reclassification of the Lophoziaceae/Scapaniaceae clade. Phytotaxa 3: 47-53.

Uotila, P. 2013. Finnish botanists on the Kola Peninsula (Russia) up to 1918. Memoranda Soc. Fauna Flora Fennica 89: 75-104.

Váňa, J., Söderström, L., Hagborg, A. \& von Konrat, M. 2012. Notes on Early Land Plants Today. 8. New combinations and some lectotypifications in Mesoptychia. Phytotaxa 6: 52-56.

Váňa, J., Söderström, L., Hagborg, A. \& von Konrat, M. 2013. Notes on Early Land Plants Today. 41. New combinations and synonyms in Cephaloziaceae (Marchantiophyta). Phytotaxa 112(1): 7-15. http:/ /dx.doi.org/10.11646/phytotaxa.112.1.2

Vilnet, A.A., Konstantinova, N.A. \& Troitsky, A.V. 2011. Taxonomical rearrangements of Solenostomataceae (Marchantiophyta) with description of a new family Endogemmataceae based on trnL-F cpDNA analysis. Folia Cryptogamica Estonica 48: 125-133.

Vilnet, A.A., Konstantinova, N.A. \& Troitsky A.V. 2012. Molecular phylogeny and systematics of the suborder Cephaloziineae with special attention to the family Cephaloziaceae s.1. (Jungermanniales, Marchantiophyta). Arctoa 21: 113-132. 
12 Folia Cryptog. Estonica 